\title{
Cellular coordination controlling organ separation and surface integrity in plants
}

\author{
Yuree Lee ${ }^{1, *}$ E June M. Kwak ${ }^{1,2, *}$ \\ ${ }^{1}$ Center for Plant Aging Research, Institute for Basic Science, Daegu 42988, ${ }^{2}$ Department of New Biology, DGIST, Daegu 42988, Korea
}

\begin{abstract}
Plants are unable to relocate themselves to a more favorable location and thus have to deal with developmental programs and environmental cues wherever they happen to be. It is yet largely unknown how plant cells coordinate cellular activities and architectures to accomplish developmental processes and respond to environmental changes. By identifying and establishing a new cellular model system, we have discovered that two neighboring cell types in the abscission zone (AZ) of Arabidopsis flowers coordinate their activities to ensure a precise "cut" through a highly restricted area of plant tissue to bring about organ separation. From this perspective, we further discuss the essence of cellular coordination in AZ, the key molecules controlling the organ separation, and relevant implications. [BMB Reports: Perspective 2018; 51(7): 317-318]
\end{abstract}

Plant organs, including leaves, fruits, and floral organs, are detached from the plant body during the developmental process. This organ separation is crucial for reproduction and adaptation of plants. Organ separation, also called abscission, mainly occurs in the pre-defined abscission zone (AZ) where cell wall linkages in the middle lamella are disconnected. Cell wall dissolution exposes the cells to dehydration and environmental perils. Thus, spatiotemporal cellular precision is the key to processing organ separation, during which cell

*Corresponding author. Yuree Lee, E-mail: yuree@ibs.re.kr; June M. Kwak, E-mail: jkwak@dgist.ac.kr

https://doi.org/10.5483/BMBRep.2018.51.7.142

Received 12 June 2018

Keywords: Abscission, Cell wall processing, Lignin molecular brace, Reactive oxygen species

Abbreviations: AZ, Abscission zone; CASP1, CASPARIAN STRIP DOMAIN PROTEIN 1; RbohF, RESPIRATORY BURST NADPH OXIDASE PROTEIN F; REC, Residuum cell; ROS, Reactive oxygen species; SEC, Secession cell

Perspective to: Yuree Lee et al. (2018) A Lignin Molecular Brace Controls Precision Processing of Cell Walls Critical for Surface Integrity in Arabidopsis. Cell 173(6): 1468-1480 doi: 10.1016/j. cell.2018.03.060 surface protection has to take place simultaneously. We have shown that the spatial coordination of cellular activities in two neighboring cell types is critical for achieving precision abscission and maintaining surface integrity (Lee et al (2018) Cell 173, 1468-1480). We have named AZ cells of the receptacle and separated organs as residuum cells (RECs) and secession cells (SECs), respectively. SECs produce a honeycomb structure of lignin which acts as a mechanical brace to localize cell wall breakdown, whereas RECs become a new epidermis by forming a protective cuticle on the cell surface.

A clue indicating how plants specify the identities of RECs and SECs comes from an investigation on reactive oxygen species (ROS). ROS accumulation in AZ displays cell type-specificity with $\mathrm{H}_{2} \mathrm{O}_{2}$ being accumulated in SECs, and $\mathrm{O}_{2} \cdot{ }^{-}$in RECs. The function of differential ROS distribution has also been shown in Arabidopsis roots where $\mathrm{O}_{2}$. accumulation was mainly in the meristematic zone and $\mathrm{H}_{2} \mathrm{O}_{2}$ was mostly accumulated in the elongation zone (Tsukagoshi et al (2010) Cell 143, 606-616). The balance between the zone-specific ROS in the cell proliferation and cell elongation zones is modulated by the transcription factor UPBEAT1 that directly regulates the expression of a set of peroxidases. It is still unclear as to how the zone-specific ROS distribution is maintained. Given the highly active and short-lived properties of ROS, it is reasonable to presume that the spatial control of ROS conversion and scavenging could contribute to the zonal specificity of ROS distribution. In this context, it is worth noting that CASP1-mediated assembly of RbohF NADPH oxidase and PEROXIDASE64 is pivotal for lignin polymerization in the Casparian strip, suggesting that ROS generation and utilization machinery are physically associated (Lee et al (2013) Cell 153, 402-412). It would be intriguing to test whether this assembly can be applied to other developmental processes requiring the spatial maintenance of ROS homeostasis.

Protection of the new cell surface while it is turning into a new epidermis is a representative function of RECs. During and after cell wall dissolution, RECs have to be protected against activities of cell wall hydrolyzing enzymes and environmental harm. RECs appear to protect their cell walls from being processed by localizing cell wall hydrolyzing enzymes inside the cells. This is in contrast to SECs, where the same proteins are secreted to the apoplastic space. Another noteworthy observation is that the diffusion of cell wall 
enzymes into RECs is limited in the lignin-deficient NADPH oxidase $r b o h D / F$ mutants in which uncontrolled cell wall digestion occurs only in SECs. It would be worthwhile to determine how RECs prevent the cell wall processing enzymes from approaching them.

Lignin is a representative characteristic of SECs and spatially restricts cell wall degrading enzymes to the site of the cell-cell detachment. To successfully accomplish the function, plants have evolved the structure of lignin in SECs. The corners of two to three layers of cells are lignified resulting in a hexagonal structure of lignin that is sturdy enough to hold each of the cells together. The top side of SECs is free of lignin, which provides open spaces for cell wall dissolution. Such a lignin-free zone is also crucial for heavily lignified tissues such as the pedicel of rice. Seed shattering is one of the primary traits that have been considered in crop domestication. Mature seeds of an Indian rice variety known as Kasalath are easily dispersed, whereas the seeds of cultivated rice Ilpum remain at the pedicel even after full maturation. The pedicel area, including AZ, is heavily lignified during seed maturation, and the existence of the lignin-free area in AZ comprises the big difference between Kasalath and Ilpum. Easy-shattering Kasalath displays a non-lignified layer in AZ surrounded by lignified pedicel cells, whereas AZ cells of non-shattering Ilpum are cemented with lignin, not allowing room for the dissolution of the cell wall (Yoon et al (2014) Plant J 79, 717-728). Further investigation of lignin functions and the underlying mechanisms regulating organ separation and seed shattering will shed light on the cellular coordination that controls plant development.

\section{ACKNOWLEDGEMENTS}

Research in the authors' laboratory was supported by IBS-R013-G2 from the Institute for Basic Science and in part by start-up funds from DGIST to J.M.K. 